\title{
Research Article \\ Some Condition for Scalar and Vector Measure Games to Be Lipschitz
}

\author{
F. Centrone ${ }^{1}$ and A. Martellotti ${ }^{2}$ \\ ${ }^{1}$ Dipartimento di Studi per l'Economia e l'Impresa, Università del Piemonte Orientale "A. Avogadro", Via Perrone 18, \\ 28100 Novara, Italy \\ ${ }^{2}$ Dipartimento di Matematica e Informatica, Università di Perugia, Via Vanvitelli 1, 06123 Perugia, Italy
}

Correspondence should be addressed to F. Centrone; francesca.centrone@eco.unipmn.it

Received 12 April 2014; Accepted 10 September 2014; Published 1 October 2014

Academic Editor: Biren N. Mandal

Copyright (c) 2014 F. Centrone and A. Martellotti. This is an open access article distributed under the Creative Commons Attribution License, which permits unrestricted use, distribution, and reproduction in any medium, provided the original work is properly cited.

We provide a characterization for vector measure games $\nu=f \circ \mu$ in $p N A_{\infty}$, with $\mu$ vector of nonatomic probability measures, analogous to the one of Tauman for games in $p N A$, and also provide a necessary and sufficient condition for a particular class of vector measure games to belong to $A C_{\infty}$.

\section{Introduction}

Measure games, that is, transferable utility (TU) games of the form $\nu=f \circ \mu$, where $\mu$ is a nonatomic measure on $\sigma$-algebra $\Sigma$ of a space $\Omega$ and $f$ is a function defined on the range of $\mu$, with $f(0)=0$, arise in several contexts including game theory, mathematical economics, and even finance (where, under suitable hypotheses on $f$ and $\mu$, they are termed as distorted probabilities). One of the reasons of their popularity lies in the fact that they generate fundamental spaces of games and that many games of interest, such as, for instance, market games of finite type, fall into this category. Classically in the literature, one distinguishes between scalar measure games where $\mu$ is a nonnegative scalar measure and vector measure games where $\mu$ is an $n$-dimensional measure with nonnegative components. The extension to signed measure is also customary.

The most classical space related to measure games is $p N A$, that is, the closed linear subspace of $B V$ generated by all powers (with respect to pointwise multiplication) of nonatomic probability measures. Several results exist, concerning scalar measure games in $p N A[1,2]$, while the only characterization of vector measure games in this space is the one of Tauman [2].
Besides the $B V$-norm, one encounters in the literature the $\|\cdot\|_{\infty}$-norm that defines the subspace $A C_{\infty} \subset B V$ of the socalled Lipschitz games. Then clearly one may define also the space $p N A_{\infty}$ as the $\|\cdot\|_{\infty}$-closure of the space generated by all powers of nonatomic probability measures. In this space, the only characterization of vector measure games we are aware of up to now is due to Milchtaich [3], and it requires the function $f$ to be continuously differentiable.

All these results put in evidence how measure games are difficult to characterize once the differentiability assumption is dropped, though economically significant measure games that do not fall into this category exist in the literature. For example, Milchtaich's characterization shows that if $f$ is piecewise linear, then measure games of the form $f \circ \mu$ do not belong to $p N A_{\infty}$; on the other side the linear span of games with $f$ piecewise linear and $\mu$ vector of mutually singular nonatomic probability measures plays a role for example in value theory [4].

In this paper, we face the problem of characterizing measure games both in $p N A_{\infty}$ and in $A C_{\infty}$. Our starting point is the characterization for scalar measure games in $A C_{\infty}$ given in [5]; there we proved that $f \circ \mu \in A C_{\infty}$ if and only if $f$ is Lipschitz. Here we introduce a generalization of the Lipschitz condition, namely, lipschitzianity in link directions, which proves to characterize vector measure 
games $f \circ \mu$ in $A C_{\infty}$, when the range of $\mu$ has finitely many exposed points and which thus covers interesting cases in literature. As a consequence, we extend the characterization in [5] to measure games of the form $f \circ \mu$ where $\mu$ is a signed measure.

Another interesting subspace is the class $B C$ of BurkillCesari $(B C)$ integrable games, introduced in [6]. The investigation on this space has been further developed in [5]; there it has been proved that the Burkill-Cesari integral is a $\|\cdot\|_{\infty}{ }^{-}$ continuous (semi)value (but in general not $B V$-continuous) and that it differs from the Aumann and Shapley value. Actually, the $B C$ integral and the space $B C$ turned out to be fruitful to provide a proper subspace of $A C$ strictly larger than $P N A$ on which a value can be defined; remember that existence and uniqueness of a value on $p N A$ are well known, while the question is still open on $A C$. In force of these results, a better understanding of the structure of the space $B C \cap$ $A C_{\infty}$, starting from its simplest elements, that is, measure games, seems to be an interesting task.

The outline of the paper is as follows. In Section 3 we characterize vector measure games in $p N A_{\infty}$; although the statement of the result is formally analogous to the one of Tauman, the proof differs from his, and this is essentially due to the difficulties arising in handling the $\|\cdot\|_{\infty}$-norm on this space. In Section 4 we first characterize a particular class of vector measure games in $A C_{\infty}$ and, as a corollary, also measure games where $\mu$ is a signed measure are characterized through lipschitzianity. The final part of the section is devoted to the investigation of $B C$ integrable Lipschitz measure games; we completely characterize the one-dimensional ones and provide a necessary condition in larger dimensions; also a topological condition is given, to ensure that a Lipschitz measure game is $\|\cdot\|_{\infty}$-close to a $B C$ integrable one.

\section{Preliminaries}

In the following we will deal with the following elements, as in [1].

$(\Omega, \Sigma)$ denotes a measurable space isomorphic to $([0,1], \mathscr{B})$ (where $\mathscr{B}$ denotes the Borel $\sigma$-algebra on $[0,1])$.

A transferable utility (TU) game $v$ is a real valued function on $\Sigma$ such that $\nu(\emptyset)=0$.

The set of all nonatomic measures on $(\Omega, \Sigma)$ is denoted by $N A$, the cone of nonnegative measures of $N A$ by $N A^{+}$, while the set of probability measures in $N A$ is indicated by $N A^{1}$. Given $\mu \in N A$, the variation measure is denoted by $|\mu|$. For a vector measure $\mu=\left(\mu_{1}, \ldots, \mu_{n}\right) \in(N A)^{n}$, the variation measure is defined by $|\mu|=\sum_{i=1}^{n}\left|\mu_{i}\right|$.

A game $v$ is said to be Lipschitz if there exists $\mu \in N A^{+}$ such that for every link $S \subset T \subset \Omega$ in $\Sigma$ it holds

$$
|\nu(T)-\nu(S)| \leq \mu(T)-\mu(S) .
$$

The space of Lipschitz games is denoted by $A C_{\infty}$ for it is a Banach space under the norm $\|\nu\|_{\infty}$ defined in the following way; for every $\mu \in N A^{+}$such that (1) holds, write $-\mu \preceq \nu \preceq \mu$. Then set

$$
\|\nu\|_{\infty}=\inf \left\{\mu(\Omega), \mu \in N A^{+},-\mu \preceq \nu \preceq \mu\right\} .
$$

To simplify notation, given a game $v \in A C_{\infty}$, and for fixed $H \in \Sigma$ we will denote as usual by $\Pi(H)$ the class of finite partitions of $H$ consisting of elements in $\Sigma$; for every $D \in \Pi(H)$, say, $D=\left\{D_{1}, \ldots, D_{k}\right\}$, let $\mathscr{T}_{D}=\left\{T_{D}=\right.$ $\left\{T_{1}, \ldots, T_{k}\right\}$ with $\left.T_{i} \subset D_{i}^{c}, T_{i} \in \Sigma\right\}$. Then denote

$$
S\left(\nu, D, T_{D}\right)=\sum_{i=1}^{k}\left[\nu\left(D_{i} \cup T_{i}\right)-v\left(T_{i}\right)\right] .
$$

For a game $\nu \in A C_{\infty}$ define, according to [7], the measures

$$
\begin{aligned}
& \nu^{*}(H)=\sup \left\{S\left(\nu, D, T_{D}\right), D \in \Pi(H), T_{D} \in \mathscr{T}_{D}\right\}, \\
& \nu_{*}(H)=\inf \left\{S\left(\nu, D, T_{D}\right), D \in \Pi(H), T_{D} \in \mathscr{T}_{D}\right\} .
\end{aligned}
$$

Then

$$
\|\nu\|_{\infty}=\sup \left\{\left|v^{*}\right|(H)+\left|\nu_{*}\right|\left(H^{c}\right), H \in \Sigma\right\} .
$$

It is clear that if $v$ is a monotone game, then $v^{*}, v_{*}$ are nonnegative and in this case $\|\nu\|_{\infty}=\nu^{*}(\Omega)$.

Note that for every $H \in \Sigma, D \in \Pi(H), T_{D} \in \mathscr{T}_{D}$ one has

$$
\left|S\left(\nu, D, T_{D}\right)\right| \leq\|v\|_{\infty} \text {. }
$$

On the other side, since $\nu^{*}, v_{*}$ are measures, there are Hahn decompositions $\left(P_{1}, N_{1}\right),\left(P_{2}, N_{2}\right)$ which allow us to rewrite (5) as

$$
\begin{gathered}
\|\nu\|_{\infty}=\sup \left\{v^{*}\left(E \cap P_{1}\right)-v^{*}\left(E \cap N_{1}\right)+v_{*}\left(E^{c} \cap P_{2}\right)\right. \\
\left.-v_{*}\left(E^{c} \cap N_{2}\right), E \in \Sigma\right\} .
\end{gathered}
$$

Hence, if for a game $\nu$ one has $\left|S\left(\nu, D, T_{D}\right)\right| \leq \varepsilon$ for each $D \in$ $\Pi(H), T_{D} \in \mathscr{T}_{D}$ and every $H \in \Sigma$, then clearly $\|\nu\|_{\infty} \leq 4 \varepsilon$.

Given a vector measure $\mu=\left(\mu_{1}, \ldots, \mu_{n}\right) \in\left(N A^{+}\right)^{n}$ and denoting its range by $R(\mu)$, a vector measure game is a game $\nu$ of the form $\nu=f \circ \mu$, where $f$ is a real valued function defined on the range of $\mu$ and $f(0)=0$. In the case $n=1$, this type of games is called scalar measure games.

Analogously to what is usually done in the space $B V$, the symbol $p N A_{\infty}$ denotes the $\|\cdot\|_{\infty}$-closure of the space generated by all powers of nonatomic probability measures.

Given a convex subset $X$ of $\mathbb{R}^{n}$, a vector $z$ is called $X$ admissible if $z=x-y$ for some $x, y \in X$. A real valued function $f$ defined on $X$ is said to be continuously differentiable on $X$ if for each $X$ admissible $z$ the derivative $d f(x+h z) / d h$ exists at each point in the relative interior of $X$ and it can be continuously extended at each point of $X$. The space of continuously differentiable functions on a set $X$ will be denoted by $\mathscr{C}^{1}(X)$.

Given a convex compact subset $K$ of $\mathbb{R}^{n}$, a point $x \in K$ is said to be exposed if $\{x\}$ is the intersection of $K$ with some supporting hyperplane of $K$.

As in [8], given a monotone nonatomic game $\lambda$, one defines the mesh of a partition $D$ as

$$
\delta_{\lambda}(D)=\max \{\lambda(I), I \in D\} .
$$

A game $v$ is Burkill-Cesari $(B C)$ integrable with respect to $\delta_{\lambda}$ if the following limit exists, for each $E \in \Sigma$ :

$$
E \longmapsto \int_{E} v=\lim _{\substack{\delta_{\lambda}(D) \rightarrow 0 \\ D \in \Pi(E)}} \sum_{I \in D} v(I)
$$


We denote by $B C$ the space of games $v$ such that there exists $\lambda \in N A^{+}$so that $\nu$ is $B C$ integrable with respect to the mesh $\delta_{\lambda}$.

The $B C$ integral does not depend upon the integration mesh (see Proposition 5.2 in [6]); in other words, for every $\lambda \in N A^{+}$such that $\nu$ is $\delta_{\lambda}-B C$ integrable, the $B C$ integral remains the same. Moreover, the $B C$ integral of a game $v$ is a finitely additive measure.

\section{Measure Games in $p N A_{\infty}$}

In [5] we have obtained a characterization (Proposition 12) of scalar measure games (made through a nonnegative measure) for the whole space $A C_{\infty}$. Anyway the Lipschitz condition on $f$ is not sufficient to ensure that a measure game belongs to $p N A_{\infty}$, that is, to the $\|\cdot\|_{\infty}$-closure of the space generated by all powers of nonatomic probability measures. Indeed, in [3] Milchtaich has shown that continuous differentiability is required, and it guarantees that a vector measure game belongs to $p N A_{\infty}$. To our knowledge, this is also the unique characterization of vector measure games in $p N A_{\infty}$ so far. It is well known that $p N A_{\infty}$ is strictly contained in $p N A$ and that many games of interest belong to this space [4].

Our first goal is to obtain a characterization for vector measure games in $p N A_{\infty}$, similar to the one given by Tauman for $p N A$ [2]. We point out that the technique of the proof is different, due to the difficulties in dealing with approximations in the $\|\cdot\|_{\infty}$-norm instead of in the $B V$ norm.

We begin with a Lemma.

Lemma 1. Let $\mu \in\left(N A^{1}\right)^{n}, f \in \mathscr{C}^{1}([R(\mu)])$. Then for every $\varepsilon>0$ there exists a polynomial in $n$ variables $p$ such that

$$
\|(f \circ \mu)-(p \circ \mu)\|_{\infty}<\varepsilon .
$$

Proof. According to [1] for every $\varepsilon>0$ there exists a polynomial $p$ on $R(\mu)$ such that the norm $\|f-p\|_{I}<\varepsilon$, where one defines

$$
\|f-p\|_{I}=\|f-p\|_{u}+\sum_{i=1}^{n}\left\|\frac{\partial f}{\partial x_{i}}-\frac{\partial p}{\partial x_{i}}\right\|_{u}
$$

and $\|\cdot\|_{u}$ is the usual uniform norm on continuous functions.

Without loss of generality we assume $p(0)=0$.

Fix $H \in \Sigma, D \in \Pi(H), T_{D} \in \mathscr{T}_{D}$. From the Mean Value Theorem we have the following:

$$
\begin{aligned}
& \left|S\left[(p \circ \mu)-(f \circ \mu), D, T_{D}\right]\right| \\
& \leq \sum_{i} \mid\left[(p \circ \mu)\left(D_{i} \cup T_{i}\right)-(f \circ \mu)\left(D_{i} \cup T_{i}\right)\right] \\
& \quad-\left[(p \circ \mu)\left(T_{i}\right)-(f \circ \mu)\left(T_{i}\right)\right] \mid \\
& =\sum_{i}\left|\nabla(f-p)\left[c_{i} \mu\left(D_{i} \cup T_{i}\right)+\left(1-c_{i}\right) \mu\left(T_{i}\right)\right] \cdot \mu\left(D_{i}\right)\right| \\
& \leq \sum_{i} \sum_{j=1}^{n}\left|\left(\frac{\partial f}{\partial x_{j}}-\frac{\partial p}{\partial x_{j}}\right)\left[c_{i} \mu\left(D_{i} \cup T_{i}\right)+\left(1-c_{i}\right) \mu\left(T_{i}\right)\right]\right| \\
& \quad \times \mu_{j}\left(D_{i}\right) .
\end{aligned}
$$

Now $c_{i} \mu\left(D_{i} \cup T_{i}\right)+\left(1-c_{i}\right) \mu\left(T_{i}\right) \in R(\mu)$ for each $i=1, \ldots, n$ and hence

$$
\begin{aligned}
& \left|\left(\frac{\partial f}{\partial x_{j}}-\frac{\partial p}{\partial x_{j}}\right)\left[c_{i} \mu\left(D_{i} \cup T_{i}\right)+\left(1-c_{i}\right) \mu\left(T_{i}\right)\right]\right| \\
& \quad \leq\left\|\frac{\partial f}{\partial x_{j}}-\frac{\partial p}{\partial x_{j}}\right\|_{u}<\varepsilon .
\end{aligned}
$$

Thus

$$
\begin{aligned}
& \left|S\left[(p \circ \mu)-(f \circ \mu), D, T_{D}\right]\right| \\
& \quad \leq \varepsilon \sum_{i} \sum_{j} \mu_{j}\left(D_{i}\right)=\varepsilon \sum_{j} \mu_{j}(H)=\varepsilon|\mu|(H) .
\end{aligned}
$$

Tauman [2] gave the definition of $\|\cdot\|_{B V}$-continuity at $\mu$ for a function $f$ defined on the range of a vector measure $\mu \epsilon$ $\left(N A^{1}\right)^{n}$, which we recall below.

Given $\mu \in(N A)^{n}$, denote with $B(\mu)$ the set of measures $\eta \in(N A)^{n}$ having the same range of $\mu$ and, for $\varepsilon>0$, define

$$
B(\mu, \varepsilon)=\{\eta \in B(\mu) \text { such that }|\eta-\mu|(\Omega)<\varepsilon\} .
$$

Fixed $\mu \in\left(N A^{1}\right)^{n}$, a function $f$ on $R(\mu)$ (with $f(0)=$ 0 ), is $\|\cdot\|_{B V}$-continuous at $\mu$, if for every $\varepsilon>0$ there exists $\delta>0$ such that for each $\eta \in B(\mu, \delta)$ there follows $\|(f \circ \mu)-(f \circ \eta)\|_{B V}<\varepsilon$. Tauman proved that a vector measure game $f \circ \mu, \mu \in\left(N A^{1}\right)^{n}$, belongs to $p N A$ if and only if $f$ is $\|\cdot\|_{B V^{-}}$continuous at $\mu$.

We translate the same definition using the $\|\cdot\|_{\infty}$-norm; that is, we say that a function $f$ on $R(\mu)$ is $\|\cdot\|_{\infty}$-continuous at $\mu$ if for every $\varepsilon>0$ there exists $\delta>0$ such that for each $\eta \in$ $B(\mu, \delta)$ there follows $\|(f \circ \mu)-(f \circ \eta)\|_{\infty}<\varepsilon$. Then we are able to prove that the analogous characterization of Tauman holds for vector measure games in $p N A_{\infty}$.

Theorem 2. $f$ is $\|\cdot\|_{\infty}$-continuous at $\mu \in\left(N A^{1}\right)^{n}$ if and only if $(f \circ \mu) \in p N A_{\infty}$.

Proof. We first prove the sufficient condition; namely, we assume that $f$ is $\|\cdot\|_{\infty}$-continuous at $\mu \in\left(N A^{1}\right)^{n}$.

Note first that without loss of generality we can assume that $R(\mu)$ has nonempty interior.

As in [2], fix $a>0$ such that the cube

$$
C=\frac{\mu(\Omega)}{2}+\left[-\frac{a}{2}, \frac{a}{2}\right]^{n} \subset R^{o}(\mu),
$$

and for $\delta \in] 0,1[$ define

$$
f^{\delta}(x)=\frac{1}{a^{n}} \int_{C} f[(1-\delta) x+\delta y] d y-\frac{1}{a^{n}} \int_{C} f(\delta y) d y .
$$

Thus $f^{\delta}(0)=0$ and according to [2, Lemma 7] $f^{\delta} \in$ $\mathscr{C}^{1}[R(\mu)]$.

We also need the following structure Lemma [2, Lemma 9]. 
Lemma 3. Let $\eta \in\left(N A^{1}\right)^{n}$ and let $\Omega_{1} \subset \Omega_{2} \subset \Omega$ be a link with both $\Omega_{1}, \Omega \backslash \Omega_{2}$ uncountable. Then, for every $\left.\delta \in\right] 0,1[$ and for every $y \in R(\eta)$ there exists $\eta_{y} \in B(\eta, 2 n \delta)$ with $\eta_{y}(H)=$ $(1-\delta) \eta(H)+\delta y$ for each $H \in \Sigma$ with $\Omega_{1} \subset H \subset \Omega_{2}$.

Let $\delta$ be momentarily fixed, and let $\{T, S \cup T\}$ be any link in $\Sigma$, with $T, \Omega \backslash(S \cup T)$ uncountable. Consider the difference $\gamma=v_{\delta}-\nu$ where $v_{\delta}=f^{\delta} \circ \mu, \nu=f \circ \mu$. We have

$$
\begin{aligned}
& \gamma(S \cup T)-\gamma(T) \\
& =f^{\delta}[\mu(S \cup T)]-f[\mu(S \cup T)]-f^{\delta}[\mu(T)]+f[\mu(T)] \\
& =\frac{1}{a^{n}} \int_{C} f[(1-\delta) \mu(S \cup T)+\delta y] d y \\
& \quad-f[\mu(S \cup T)]-\frac{1}{a^{n}} \int_{C} f[(1-\delta) \mu(T)+\delta y] d y \\
& \quad+f[\mu(T)] \\
& =\frac{1}{a^{n}} \int_{C}\{f[(1-\delta) \mu(S \cup T)+\delta y]-f[\mu(S \cup T)] \\
& \quad \quad-f[(1-\delta) \mu(T)+\delta y]+f[\mu(T)]\} d y .
\end{aligned}
$$

Now by Lemma 3 applied with $T=\Omega_{1}, S \cup T=\Omega_{2}$, and $\eta=\mu$ for every $y \in C$ there is $\eta_{y} \in B(\mu, 2 n \delta)$ such that $\eta_{y}(S \cup T)=(1-\delta) \mu(S \cup T)+\delta y$ and $\eta_{y}(T)=(1-\delta) \mu(T)+\delta y$. Hence, continuing the computation (18) we reach

$$
\begin{aligned}
\gamma(S \cup T)-\gamma(T) \\
=\frac{1}{a^{n}} \int_{C}\left\{\left[\left(f \circ \eta_{y}\right)(S \cup T)-(f \circ \mu)(S \cup T)\right]\right. \\
\left.-\left[\left(f \circ \eta_{y}\right)(T)-(f \circ \mu)(T)\right]\right\} d y .
\end{aligned}
$$

By the assumption corresponding to $\varepsilon>0$ there exists $\delta_{o}>0$ such that for $m \in B\left(\mu, \delta_{o}\right)$ one has $\|(f \circ m)-(f \circ \mu)\|_{\infty}<\varepsilon$.

Let us now fix $\delta<\delta_{o} / 2 n$, and let $H \in \Sigma, D \in \Pi(H), T_{D} \in$ $\mathscr{T}_{D}$ be fixed also, in such a way that $T_{i}, \Omega \backslash\left(D_{i} \cup T_{i}\right)$ are uncountable.

Then we have

$$
\begin{aligned}
& S\left(\gamma, D, T_{D}\right) \\
& =\sum_{i} \gamma\left(D_{i} \cup T_{i}\right)-\gamma\left(T_{i}\right) \\
& =\frac{1}{a^{n}} \sum_{i} \int_{C}\left\{\left[\left(f \circ \eta_{y}\right)\left(D_{i} \cup T_{i}\right)-(f \circ \mu)\left(D_{i} \cup T_{i}\right)\right]\right. \\
& \left.\quad-\left[\left(f \circ \eta_{y}\right)\left(T_{i}\right)-(f \circ \mu)\left(T_{i}\right)\right]\right\} d y \\
& =\frac{1}{a^{n}} \int_{C} S\left\{\left[\left(f \circ \eta_{y}\right)-(f \circ \mu)\right], D, T_{D}\right\} d y .
\end{aligned}
$$

Since $\eta_{y} \in B(\mu, 2 n \delta) \subset B\left(\mu, \delta_{o}\right)$ we know that $\|\left(f \circ \eta_{y}\right)-$ $(f \circ \mu) \|_{\infty}<\varepsilon$, and from $(6),\left|S\left\{\left[\left(f \circ \eta_{y}\right)-(f \circ \mu)\right], D, T_{D}\right\}\right|<$ $\varepsilon$ for each $y \in[0,1]$. Hence $\left|S\left(\gamma, D, T_{D}\right)\right|<\varepsilon$ for each pair $D \in \Pi(H), T_{D} \in \mathscr{T}_{D}$ that satisfies the cardinality conditions at each link that proves that $f^{\delta} \circ \mu$ approximates $f \circ \mu$ in the $\|\cdot\|_{\infty}$-norm.

To conclude our proof, we will prove that for every $H \in$ $\Sigma, D \in \Pi(H), T_{D} \in \mathscr{T}_{D}$ can be replaced by a choice $H^{\sharp} \in$ $\Sigma, D^{\sharp} \in \Pi\left(H^{\sharp}\right), T_{D}^{\sharp} \in \mathscr{T}_{D}$ so that each link $\left(T_{i}^{\sharp}, D_{i}^{\sharp} \cup T_{i}^{\sharp}\right)$ fulfills the cardinality requirements for each link, and $S\left[\gamma, D, T_{D}\right]=$ $S\left[\gamma, D^{\sharp}, T_{D^{\sharp}}^{\sharp}\right]$.

To this aim, let $\varepsilon, \delta, H \in \Sigma, D \in \Pi(H), T_{D} \in \mathscr{T}_{D}$ be fixed, with $D=\left\{D_{1}, \ldots, D_{k}\right\}$. Without loss of generality we can assume that each $D_{i}$ is not $\mu$-null.

Let $I \subset\{1, \ldots, k\}$ be the set of indexes for which the corresponding link $\left(T_{i}, D_{i} \cup T_{i}\right)$ does not fulfill the cardinality requirements; then $I$ can be split into two disjoint subsets $I_{1}$ and $I_{2}$, where $I_{1}$ is the set of indexes for which $\Omega \backslash\left(D_{i} \cup T_{i}\right)$ is at most countable.

For each $i \in I_{2}$ one can choose an uncountable $\mu$-null set $N_{i}$ in $\Omega \backslash\left(D_{i} \cup T_{i}\right)$ and then replace $T_{i}$ with $\widetilde{T}_{i}=T_{i} \cup N_{i}$; since $i \in I_{2}$, we have $T_{i}$ countable, and, therefore, $\mu\left(T_{i}\right)=0$; thus the replacement does not affect the corresponding summand in $S\left(\gamma, D, T_{D}\right)$.

For each index $i \in I_{1}$ choose an uncountable $\mu$-null set $N_{i} \subset D_{i}$ and replace $D_{i}$ with $\widetilde{D_{i}}=D_{i} \backslash N_{i}, T_{i}$ with $\widetilde{T}_{i}=T_{i} \cup N_{i}$.

Then we can replace $D$ with $D^{\sharp}=\left\{D_{i}^{\sharp}, i=1, \ldots, n\right\}$ setting

$$
D_{i}^{\sharp}= \begin{cases}\widetilde{D_{i}} & \text { if } i \in I_{1}, \\ D_{i} & \text { otherwise }\end{cases}
$$

and analogously $T_{D}$ with $T_{D}^{\sharp}=\left\{T_{i}^{\sharp}, i=1, \ldots, n\right\}$ where

$$
T_{i}^{\sharp}= \begin{cases}\widetilde{T}_{i} & \text { if } i \in I, \\ T_{i} & \text { otherwise }\end{cases}
$$

and finally set $H^{\sharp}=\bigcup_{i=1}^{k} D_{i}^{\sharp}$. By construction then $S\left(\gamma, D, T_{D}\right)=S\left(\gamma, D^{\sharp}, T_{D^{\sharp}}^{\sharp}\right)$.

To prove the converse implication, according to $[1$, Lemma 7.2 page 41], it is enough to prove that monomials of the form $c \mu^{\alpha}$, with $c \in \mathbb{R}, \mu \in N A^{1}, \alpha \in \mathbb{N}^{+}$, are $\|\cdot\|_{\infty^{-}}$ continuous at each $\mu \in N A^{1}$.

We first prove that if $\mu \in B(m, \delta), H \in \Sigma, D=\left\{D_{i}\right\}_{i=1, \ldots, k} \in$ $\Pi(H), p \in \mathbb{N}$, then

$$
\sum_{i=1}^{k}\left|m^{p}\left(D_{i}\right)-\mu^{p}\left(D_{i}\right)\right|<p \delta .
$$

In fact, for each summand we have

$$
\begin{aligned}
\left|m^{p}\left(D_{i}\right)-\mu^{p}\left(D_{i}\right)\right| \leq & m^{p-1}\left(D_{i}\right)\left|m\left(D_{i}\right)-\mu\left(D_{i}\right)\right| \\
& +\mu\left(D_{i}\right)\left|m^{p-1}\left(D_{i}\right)-\mu^{p-1}\left(D_{i}\right)\right| \\
\leq & m\left(D_{i}\right)|m-\mu|\left(D_{i}\right)+\mu\left(D_{i}\right)(p-1) \delta,
\end{aligned}
$$

where the last inequality follows from Lipschitz condition on $[0,1]$ and the fact that $m \in N A^{1}$. Then in $B(m, \delta)$ we have further $\left|m^{p}\left(D_{i}\right)-\mu^{p}\left(D_{i}\right)\right|<\delta m\left(D_{i}\right)+(p-1) \mu\left(D_{i}\right) \delta$, whence for the whole sum we obtain (23). 
Let now as usual $H \in \Sigma, D \in \Pi(H), D=\left\{D_{i}\right\}, T_{D} \in \mathscr{T}_{D}$ be fixed. Consider the single summand in $S\left(m^{\alpha}-\mu^{\alpha}, D, T_{D}\right)$

$$
\begin{aligned}
& m^{\alpha}\left(T_{i} \cup D_{i}\right)-\mu^{\alpha}\left(T_{i} \cup D_{i}\right)-m^{\alpha}\left(T_{i}\right)+\mu^{\alpha}\left(T_{i}\right) \\
& =m^{\alpha}\left(D_{i}\right)-\mu^{\alpha}\left(D_{i}\right) \\
& \quad+\sum_{j=1}^{\alpha-1}\left(\begin{array}{c}
\alpha \\
j
\end{array}\right)\left[m^{\alpha-j}\left(D_{i}\right) m^{j}\left(T_{i}\right)-\mu^{\alpha-j}\left(D_{i}\right) \mu^{j}\left(T_{i}\right)\right] .
\end{aligned}
$$

In the sum $S\left(m^{\alpha}-\mu^{\alpha}, D, T_{D}\right)$ there will then appear a sum of the form in (23) which we can estimate with $\alpha \delta$.

Let us now treat the summands of the form $\left[m^{\alpha-j}\left(D_{i}\right) m^{j}\left(T_{i}\right)-\mu^{\alpha-j}\left(D_{i}\right) \mu^{j}\left(T_{i}\right)\right]$. Clearly

$$
\begin{aligned}
m^{\alpha-j} & \left(D_{i}\right) m^{j}\left(T_{i}\right)-\mu^{\alpha-j}\left(D_{i}\right) \mu^{j}\left(T_{i}\right) \\
= & m^{\alpha-j}\left(D_{i}\right)\left[m^{j}\left(T_{i}\right)-\mu^{j}\left(T_{i}\right)\right] \\
& +\mu^{j}\left(T_{i}\right)\left[m^{\alpha-j}\left(D_{i}\right)-\mu^{\alpha-j}\left(D_{i}\right)\right]
\end{aligned}
$$

and so in the whole $\left|S\left(m^{\alpha}-\mu^{\alpha}, D, T_{D}\right)\right|$ we can apply the following bound from above:

$$
\begin{gathered}
\sum_{i}\left|m^{\alpha-j}\left(D_{i}\right) m^{j}\left(T_{i}\right)-\mu^{\alpha-j}\left(D_{i}\right) \mu^{j}\left(T_{i}\right)\right| \\
\leq \sum_{i} m^{\alpha-j}\left(D_{i}\right)\left|m^{j}\left(T_{i}\right)-\mu^{j}\left(T_{i}\right)\right| \\
+\mu^{j}\left(T_{i}\right)\left|m^{\alpha-j}\left(D_{i}\right)-\mu^{\alpha-j}\left(D_{i}\right)\right| .
\end{gathered}
$$

Now each term $\left|m^{j}\left(T_{i}\right)-\mu^{j}\left(T_{i}\right)\right| \leq j \delta$ again by Lipschitz condition and the choice of $m \in B(\mu, \delta)$. Also $m^{\alpha-j}\left(D_{i}\right) \leq$ $m\left(D_{i}\right)$ since $m \in N A^{1}$ and $\mu\left(T_{i}\right) \leq 1$ for the same reason. Hence, because of (23),

$$
\begin{aligned}
& \sum_{i}\left|m^{\alpha-j}\left(D_{i}\right) m^{j}\left(T_{i}\right)-\mu^{\alpha-j}\left(D_{i}\right) \mu^{j}\left(T_{i}\right)\right| \\
& \quad \leq j \delta \sum_{i} m\left(D_{i}\right)+\sum_{i}\left|m^{\alpha-j}\left(D_{i}\right)-\mu^{\alpha-j}\left(D_{i}\right)\right| \\
& \quad<j \delta+(\alpha-j) \delta=\alpha \delta .
\end{aligned}
$$

Finally

$$
\begin{aligned}
& \sum_{i} \sum_{j=1}^{\alpha-1}\left(\begin{array}{c}
\alpha \\
j
\end{array}\right)\left|m^{\alpha-j}\left(D_{i}\right) m^{j}\left(T_{i}\right)-\mu^{\alpha-j}\left(D_{i}\right) \mu^{j}\left(T_{i}\right)\right| \\
& =\sum_{j=1}^{\alpha-1}\left(\begin{array}{c}
\alpha \\
j
\end{array}\right) \sum_{i}\left|m^{\alpha-j}\left(D_{i}\right) m^{j}\left(T_{i}\right)-\mu^{\alpha-j}\left(D_{i}\right) \mu^{j}\left(T_{i}\right)\right| \\
& \leq\left(2^{\alpha}-1\right) \alpha \delta
\end{aligned}
$$

from which we conclude according to (25) and (23)

$$
\left|S\left(m^{\alpha}-\mu^{\alpha}, D, T_{D}\right)\right| \leq \alpha 2^{\alpha} \delta
$$

which only depends upon $\alpha$. Hence the proof is complete.
Note that, in proving the "if" part of the above theorem, we have incidentally proven that, for a measure game $f$ 。 $\mu \in p N A_{\infty}$, for each $\varepsilon>0$ there exists $\delta>0$ such that $\left\|(f \circ \mu)-\left(f^{\delta} \circ \mu\right)\right\|_{\infty}<\varepsilon$.

On the other side, as $f^{\delta} \in \mathscr{C}^{1}[R(\mu)]$, we can apply Lemma 1 and find a polynomial $p_{\varepsilon}$ such that $\left\|\left(f^{\delta} \circ \mu\right)-\left(p_{\varepsilon} \circ \mu\right)\right\|_{\infty}<\varepsilon$.

In other words we have proven the following statement.

Theorem 4. Let $\mu \in\left(N A^{1}\right)^{n}, f: R(\mu) \rightarrow \mathbb{R}$. The following are equivalent.

(i) $(f \circ \mu)$ is in $p N A_{\infty}$;

(ii) for each $\varepsilon>0$ there exists a polynomial $p: \mathbb{R}^{n} \rightarrow \mathbb{R}$ vanishing at 0 such that

$$
\|(f \circ \mu)-(p \circ \mu)\|_{\infty}<\varepsilon .
$$

\section{Measure Games in $A C_{\infty}$}

Throughout this section we will deal with maps $f: \mathbb{R}_{+}^{n} \rightarrow \mathbb{R}$ with $f(0)=0$, namely, admissible maps.

For two vectors $\mathbf{x}, \mathbf{y} \in \mathbb{R}_{+}^{n}$ we will adopt the notation $\mathbf{x} \ll \mathbf{y}$ for the usual componentwise order. We introduce the following definitions, which extend Lipschitz condition when $n>1$.

Definition 5. The map $f$ is said to be Lipschitz in increasing directions when $L>0$ exists such that for each pair $\mathbf{x} \leq \mathbf{y}$ in $\mathbb{R}_{+}^{n}$ one has

$$
|f(\mathbf{x})-f(\mathbf{y})| \leq L \cdot\|\mathbf{x}-\mathbf{y}\| .
$$

Definition 6. Let $\mu: \Sigma \rightarrow \mathbb{R}_{+}^{n}$ be a measure; a pair $\mathbf{x}, \mathbf{y} \in R(\mu)$ is said to be a $\mu$-link direction when there exists a link $(S, S \cup T)$ in $\Sigma$ such that $\mu(S)=\mathbf{x}, \mu(S \cup T)=\mathbf{y}$.

A map $f: \mathbb{R}_{+}^{n} \rightarrow \mathbb{R}$ is then said to be Lipschitz in $\mu$ link directions if there exists $L>0$ such that for every $\mu$-link direction $\mathbf{x}, \mathbf{y}$ there holds

$$
|f(\mathbf{x})-f(\mathbf{y})| \leq L \cdot\|\mathbf{x}-\mathbf{y}\| .
$$

Note that if $f$ is Lipschitz in increasing directions, then it is Lipschitz in $\mu$-link directions for each $n$-dimensional nonnegative measure $\mu$.

Let now $n \in \mathbb{N}^{+}$and $\mu \in\left(N A^{+}\right)^{n}$ be fixed, and consider the spaces

(i) $\mathscr{A}=\left\{f: \mathbb{R}_{+}^{n} \rightarrow \mathbb{R}\right.$ admissible and Lipschitz in the increasing directions $\}$,

(ii) $\mathscr{L}(\mu)=\left\{f: \mathbb{R}_{+}^{n} \rightarrow \mathbb{R}\right.$ admissible and Lipschitz in $\mu$-link directions\}.

Note that if $f \in \mathscr{A}$, then $f \circ \mu \in A C_{\infty}$ for every $\mu \in\left(N A^{+}\right)^{n}$, while we can assert the same for $f \in \mathscr{L}(\mu)$ only for precisely $\mu$.

Now, given $f, \mu$, let $\nu=f \circ \mu$ and define the subsets of $N A^{+}$. Consider

$$
\begin{aligned}
& A(\nu)=\left\{\lambda \in N A^{+} \lambda \ll|\mu|,-\lambda \preceq \nu \preceq \lambda\right\}, \\
& C(\mu)=\left\{\lambda \ll|\mu|: \frac{d \lambda}{d|\mu|} \in L^{\infty}(|\mu|)\right\} .
\end{aligned}
$$


Note that if $\nu$ is a vector measure game in $A C_{\infty}$, then $A(\nu) \neq \emptyset$. Indeed, let $\lambda \in N A^{+}$be such that $-\lambda \preceq \nu \preceq \lambda$ and consider the Jordan decomposition $\lambda=\lambda_{1}+\lambda_{2}$ with $\lambda_{1} \ll|\mu|, \lambda_{2} \perp|\mu|$. Then there are two disjoint sets $\Omega_{1}, \Omega_{2}$ which support $\lambda_{1}, \lambda_{2}$, respectively. This means that $\mu$ vanishes on $\Omega_{2}$; hence, $\lambda_{1} \in$ $A(\nu)$.

In fact, for each link $S \subset T$, setting $S_{1}=S \cap \Omega_{1}, T_{1}=$ $T \cap \Omega_{1}$ since $\mu\left(S_{1}\right)=\mu(S), \mu\left(T_{1}\right)=\mu(T)$ one has

$$
\begin{aligned}
|\nu(T)-\nu(S)| & =\left|\nu\left(T_{1}\right)-\nu\left(S_{1}\right)\right| \leq \lambda\left(T_{1}\right)-\lambda\left(S_{1}\right) \\
& =\lambda_{1}\left(T_{1}\right)-\lambda_{1}\left(S_{1}\right)=\lambda_{1}(T)-\lambda_{1}(S) .
\end{aligned}
$$

We can now state the following.

Proposition 7. Let $\mu \in\left(N A^{+}\right)^{n}$ and $\nu=f \circ \mu$. The following are equivalent:

(i) $f \in \mathscr{L}(\mu)$;

(ii) there exists $c>0$ such that $c|\mu| \in A(\nu)$;

(iii) $A(\nu) \cap C(\mu) \neq \emptyset$.

Proof. (i) implies (ii), since, if $f \in \mathscr{L}(\mu)$, then immediately $L|\mu| \in A(\nu) \cap C(\mu)$.

The implication (ii) $\Rightarrow$ (iii) is immediate.

Finally, let $\lambda \in A(\nu) \cap C(\mu)$. This immediately implies that there exists $L>0$ such that $d \lambda / d|\mu| \leq L|\mu|$-a.e., and this in turn implies that $\lambda \leq L|\mu|$ on $\Sigma$. On the other side, as mentioned in Section 2, we have that $|\nu(S \cup T)-\nu(T)| \leq \lambda(S)$ for every pair of disjoint sets $S, T \in \Sigma$. Then immediately, on each $\mu$-link direction $\mathbf{x} \ll \mathbf{y}$ and every link $S, S \cup T$ such that $\mu(S)=\mathbf{x}, \mu(S \cup T)=\mathbf{y}$ one finds

$$
|f(\mathbf{x})-f(\mathbf{y})| \leq \lambda(S) \leq L|\mu|(S)=L \cdot\|\mathbf{x}-\mathbf{y}\|
$$

that says that $f \in \mathscr{L}(\mu)$.

Each of the conditions of Proposition 7 implies that $\nu \in$ $A C_{\infty}$. It is, therefore, rather natural to ask whether condition $f \in \mathscr{L}(\mu)$ characterizes vector measure games $f \circ \mu$ in $A C_{\infty}$. We already know from Proposition 12 in [5] that this is the case for $n=1$.

In the more general case, we have the following result.

Theorem 8. Let $\mu \in\left(N A^{+}\right)^{n}$ be such that its range $R(\mu)$ has only finitely many exposed points. Then a vector measure game $\nu=f \circ \mu \in A C_{\infty}$ if and only if $f \in \mathscr{L}(\mu)$.

Proof. We only need to prove that if $\nu \in A C_{\infty}$, then $f \in$ $\mathscr{L}(\mu)$.

Let $P_{1}, \ldots, P_{k}$ be the exposed points of $R(\mu)$, and let $A_{1}, \ldots, A_{k}$ be sets in $\Sigma$ such that $\mu\left(A_{i}\right)=P_{i}, i=1, \ldots, k$.

Consider the partition generated by $A_{1}, \ldots, A_{k}$, say, $D=\left\{\Omega_{1}, \ldots, \Omega_{n}\right\}$, and assume that each $\Omega_{i}$ has nonnull $\mu$ measure.

If $\Sigma_{o}$ denotes the algebra generated by $D$, we have that $\mu\left(\Sigma_{o}\right)=R(\mu)$; in fact by additivity each $P_{i} \in \mu\left(\Sigma_{o}\right)$ and from Lyapunov Theorem $\mu\left(\Sigma_{o}\right)$ is convex; hence $\mu\left(\Sigma_{o}\right)$ ว $\operatorname{co}\left\{P_{1}, \ldots, P_{n}\right\}=R(\mu)$ where the last equality is deduced from Straszewicz Theorem.
Let $\mathbf{x} \lll \mathbf{y}$ be a fixed $\mu$-link direction. Then $\mathbf{x}=\mathbf{x}_{1}+\cdots+\mathbf{x}_{n}$, $\mathbf{y}=\mathbf{y}_{1}+\cdots+\mathbf{y}_{n}$, where, for each $i, \mathbf{x}_{i}, \mathbf{y}_{i} \in\left\{t \mu\left(\Omega_{i}\right), t \in[0,1]\right\}$. Moreover, easily $\mathbf{x}_{i} \ll \mathbf{y}_{i}$.

Set now $\mathbf{x}=\mathbf{v}_{o}, \mathbf{v}_{i}=\sum_{j=1}^{i} \mathbf{y}_{j}+\sum_{j=i+1}^{n} \mathbf{x}_{j}, i=1, \ldots, n-$ $1, \mathbf{v}_{n}=\mathbf{y}$.

Fix $\lambda \in A(\nu)$ and $i=1, \ldots, n$.

We know that there are $t_{i}^{\prime} \leq t_{i}^{\prime \prime} \in[0,1]$ such that $\mathbf{x}_{i}=$ $t_{i}^{\prime} \mu\left(\Omega_{i}\right), \mathbf{y}_{i}=t_{i}^{\prime \prime} \mu\left(\Omega_{i}\right)$. Choose then $S_{i}^{\prime} \subseteq S_{i}^{\prime \prime} \subset \Omega_{i}$ such that for the $(n+1)$-dimensional measure $(\lambda, \mu)$ one has $(\lambda, \mu)\left(S_{i}^{\prime}\right)=$ $t_{i}^{\prime}(\lambda, \mu)\left(\Omega_{i}\right),(\lambda, \mu)\left(S_{i}^{\prime \prime}\right)=t_{i}^{\prime \prime}(\lambda, \mu)\left(\Omega_{i}\right)$, and let $T_{i} \subset \Omega \backslash \Omega_{i}$ with

$$
\mu\left(T_{i}\right)=\sum_{j=1}^{i-1} \mathbf{y}_{j}+\sum_{j=i+1}^{n} \mathbf{x}_{j} .
$$

This is possible for $\mathbf{x}_{j}, \mathbf{y}_{j} \in R\left(\left.\mu\right|_{\Omega_{j}}\right)$ and for $i \neq j, \Omega_{i} \cap \Omega_{j}=$ $\emptyset$.

Then $S_{i}^{\prime} \cup T_{i}, S_{i}^{\prime \prime} \cup T_{i}$ is a link and hence

$$
\left|\nu\left(S_{i}^{\prime} \cup T_{i}\right)-v\left(S_{i}^{\prime \prime} \cup T_{i}\right)\right| \leq \lambda\left(S_{i}^{\prime \prime} \backslash S_{i}^{\prime}\right)=\left(t_{i}^{\prime \prime}-t_{i}^{\prime}\right) \lambda\left(\Omega_{i}\right) .
$$

But $\mu\left(S_{i}^{\prime} \cup T_{i}\right)=\mu\left(S_{i}^{\prime}\right)+\mu\left(T_{i}\right)=\mathbf{v}_{i-1}, \mu\left(S_{i}^{\prime \prime} \cup T_{i}\right)=\mathbf{v}_{i}$. Thus (38) becomes

$$
\left|f\left(\mathbf{v}_{i}\right)-f\left(\mathbf{v}_{i-1}\right)\right| \leq\left(t_{i}^{\prime \prime}-t_{i}^{\prime}\right) \lambda\left(\Omega_{i}\right) .
$$

Now

$$
\begin{aligned}
\left\|\mathbf{v}_{i}-\mathbf{v}_{i-1}\right\| & =\left\|\mathbf{y}_{i}-\mathbf{x}_{i}\right\|=\left(t_{i}^{\prime \prime}-t_{i}^{\prime}\right)\left\|\mu\left(\Omega_{i}\right)\right\| \\
& =\left(t_{i}^{\prime \prime}-t_{i}^{\prime}\right)|\mu|\left(\Omega_{i}\right) .
\end{aligned}
$$

Thus

$$
\left|f\left(\mathbf{v}_{i}\right)-f\left(\mathbf{v}_{i-1}\right)\right| \leq \frac{\lambda\left(\Omega_{i}\right)}{|\mu|\left(\Omega_{i}\right)}\left\|\mathbf{v}_{i}-\mathbf{v}_{i-1}\right\| .
$$

Since $\mathbf{v}_{i}-\mathbf{v}_{i-1}=\mathbf{y}_{i}-\mathbf{x}_{i}$, we have that $\left\|\mathbf{v}_{i}-\mathbf{v}_{i-1}\right\| \leq\|\mathbf{y}-\mathbf{x}\|$.

In conclusion we have that

$$
|f(\mathbf{x})-f(\mathbf{y})| \leq L\|\mathbf{y}-\mathbf{x}\|
$$

with $L=n \cdot \sum_{i=1}^{n}\left(\lambda\left(\Omega_{i}\right) /|\mu|\left(\Omega_{i}\right)\right)$.

Observe that the above result includes two interesting cases: the case of $\mu_{i} \perp \mu_{j}$ for each pair of components $\mu_{i}, \mu_{j}$ of $\mu$ and the case of vector measures $\mu$ which can be represented as integral measures $\left(\mu_{1}, \mu_{1}(\varphi)\right)$ where $\varphi$ is a $(n-1)$-dimensional simple function.

Also, from Theorem 8 one derives the following corollary that generalizes Proposition 12 in [5] to the case of signed scalar measure games, namely, games of the form $\nu=f \circ \mu$ with $\mu$ signed measure.

Corollary 9. Let $\mu: \Sigma \rightarrow \mathbb{R}$ be a nonatomic signed measure, and let $f:\left[-\mu^{-}(\Omega), \mu^{+}(\Omega)\right] \rightarrow \mathbb{R}$. Then $f \circ \mu \in A C_{\infty}$ if and only if $f$ is Lipschitz on $R(\mu)$. 
Proof. Consider the nonatomic two-dimensional measure $m=\left(\mu^{+}, \mu^{-}\right)$and the function $g: \mathbb{R}^{2} \rightarrow \mathbb{R}$ defined by $g(x, y)=f(x-y)$. Thus $\nu=f \circ \mu=g \circ m$. Since $R(m)$ is a rectangle, if $v \in A C_{\infty}$, from Theorem $8 g \in \mathscr{L}(m)$, namely, a constant $L>0$ exists, such that for each $m$-link direction $\mathbf{x}, \mathbf{y}$

$$
|g(\mathbf{x})-g(\mathbf{y})| \leq L\|\mathbf{y}-\mathbf{x}\|
$$

Then $f$ is also Lipschitz with constant $L$.

So far, we have not been able to answer the question whether the condition in Theorem 8 actually characterizes vector measure games in $A C_{\infty}$.

We now turn our attention to a smaller subspace of $A C_{\infty}$. In $[5,6]$ we have introduced and studied the space $B C$ of Burkill-Cesari $(B C)$ integrable games. In particular in [5] we considered the space $Q=B C \cap A C_{\infty}$ of Lipschitz games that are indeed $B C$ integrable.

Here we will consider the subspace $\mathscr{V}$ of vector measure games in $Q$.

First of all, observe that $A C_{\infty} \backslash B C$ and $B C \backslash A C_{\infty}$ are nonempty. Indeed a scalar measure game $f \circ \mu \in A C_{\infty}$ if and only if $f$ is Lipschitz on $[0, \mu(\Omega)]$, while, according to Proposition 14 in [5], $f \circ \mu \in B C$ if and only if $f$ admits right hand side derivative at 0 .

Therefore, for instance, if $f(x)=1-\sqrt{1-x}$ and $P \in N A^{1}$, $(f \circ P) \in B C \backslash A C_{\infty}$.

On the other side, let $\mu$ be a signed measure with $\mu(\Omega)=$ 0 , and let $\nu=|\mu|$; then easily $\nu \in A C_{\infty}$.

In fact, if $S, T$ is a link, then

$$
\begin{gathered}
|\nu(T)-\nu(S)|-|| \mu(T)|-| \mu(S)|| \\
\quad \leq|\mu(T)-\mu(S)| \leq|\mu|(T \backslash S)
\end{gathered}
$$

and so $|\mu| \in A(\nu)$. On the other side, $\nu \notin B C$; indeed, the following extension of Proposition 14 in [5] can be derived from Corollary 9.

Proposition 10. Take $\mu \in N A$ and let $f:\left[-\mu^{-}(\Omega), \mu^{+}(\Omega)\right] \rightarrow$ $\mathbb{R}$. Then $f \circ \mu \in \mathscr{V}$ if and only if $f$ is Lipschitz on $R(\mu)$ and $f^{\prime}(0)$ exists.

Proof. The proof of the sufficiency goes along the same lines of the proof of Theorem 6.1 in [6].

Conversely, since $v \in A C_{\infty}$ we know from Corollary 9 that $f$ is Lipschitz; hence the ratios $f(x) / x, x \neq 0$ are bounded. Assume that $v \in B C$ but $f^{\prime}(0)$ does not exist. We have then the following cases:

(i) at least one between $f_{+}^{\prime}(0)$ and $f_{-}^{\prime}(0)$ does not exist;

(ii) $f_{-}^{\prime}(0) \neq f_{+}^{\prime}(0)$.

The first case can be treated analogously to proof of Proposition 14 in [5], simply working with a set $F \subset P$, $F \subset N$, respectively, where $(P, N)$ is a Hahn decomposition of $\Omega$.

Assume then that $f_{-}^{\prime}(0)=\ell_{1} \neq \ell_{2}=f_{+}^{\prime}(0)$.
Let then $x_{n} \downarrow 0$ and let us fix $F \in \sum$ with $\mu^{+}(F)=\mu^{-}(F)>$ 0 , so that $\mu(F)=0$. Choose $\left.\varepsilon \in] 0, \mu^{+}(F)\right]$. Then there exists $\bar{n} \in \mathbb{N}$ such that for each $n>\bar{n}$

$$
\begin{gathered}
\left|\frac{f\left(x_{n}\right)}{x_{n}}-\ell_{1}\right|<\frac{\varepsilon}{3 \mu^{+}(F)}, \\
\left|\frac{f\left(-x_{n}\right)}{-x_{n}}-\ell_{2}\right|<\frac{\varepsilon}{3 \mu^{-}(F)} .
\end{gathered}
$$

By means of the continuity of $f$ at 0 , choose next $\tilde{n}>\bar{n}$ such that $|f(x)|<\varepsilon / 3$ whenever $x \leq x_{\tilde{n}}$; also $\widetilde{n}$ can be chosen so that $\left|\ell_{i}\right|\left|x_{\tilde{n}}\right|<\varepsilon / 3$ and such that $x_{\tilde{n}}\left(\lambda(F) / \mu^{+}(F)\right)<\delta(\varepsilon / 3)$ where $\delta$ is the parameter of $\delta_{\lambda} B C$ integrability.

Choose now the following $D \in \Pi(F \cap P)$; by means of Lyapunov Theorem, divide $F$ into finitely many sets, say $I_{1}, \ldots, I_{k}$, each with $(\mu, \lambda)\left(I_{j}\right)=\left(x_{\tilde{n}},\left(\lambda(F) / \mu^{+}(F)\right) x_{\tilde{n}}\right)$, until $\mu\left((F \cap P) \backslash \bigcup_{j=1}^{k} I_{j}\right) \leq x_{\tilde{n}}$ and then choose $I_{k+1}=(F \cap P) \backslash$ $\bigcup_{j=1}^{k} I_{j}$; thus easily $\lambda\left(I_{k+1}\right)=\left(\lambda(F) / \mu^{+}(F)\right) \mu\left(I_{k+1}\right)$ for

$$
\begin{aligned}
\lambda\left(I_{k+1}\right) & =\lambda(F)-\sum_{j=1}^{k} \lambda\left(I_{j}\right)=\lambda(F)-k \frac{\lambda(F)}{\mu^{+}(F)} x_{\tilde{n}} \\
& =\frac{\lambda(F)}{\mu^{+}(F)}\left[\mu^{+}(F)-k x_{\tilde{n}}\right]=\frac{\lambda(F)}{\mu^{+}(F)} \mu\left(I_{k+1}\right) .
\end{aligned}
$$

Then for $D=\left\{I_{1}, \ldots, I_{k}, I_{k+1}\right\}$ one has $\delta_{\lambda}(D)<\delta(\varepsilon / 3)$.

We have then

$$
\begin{aligned}
& \sum_{I \in D}\left|f[\mu(I)]-\ell_{1} \mu(I)\right| \\
& \quad=\sum_{n=1}^{k}\left|f\left(x_{\tilde{n}}\right)-\ell_{1} x_{\tilde{n}}\right|+\left|f\left[\mu\left(I_{k+1}\right)\right]-\ell_{1} \mu\left(I_{k+1}\right)\right| \\
& \quad \leq \sum_{n=1}^{k}\left|f\left(x_{\tilde{n}}\right)-\ell_{1} x_{\tilde{n}}\right|+\left|f\left[\mu\left(I_{k+1}\right)\right]\right|+\left|\ell_{1}\right| x_{\tilde{k}} \\
& \quad=\sum_{n=1}^{k}\left|\frac{f\left(x_{\tilde{n}}\right)-\ell_{1} x_{\tilde{n}}}{x_{\tilde{n}}}\right| x_{\tilde{n}}+\frac{\varepsilon}{3}+\frac{\varepsilon}{3} .
\end{aligned}
$$

As for the first sum we have the following estimate:

$$
\begin{aligned}
\sum_{n=1}^{k}\left|\frac{f\left(x_{\tilde{n}}\right)-\ell_{1} x_{\tilde{n}}}{x_{\tilde{n}}}\right| x_{\widetilde{n}} & <\frac{\varepsilon}{3 \mu^{+}(F)} \sum_{n=1}^{k} x_{\widetilde{n}} \\
& =\frac{\varepsilon}{3} \cdot \frac{\mu\left((F \cap P) \backslash I_{k+1}\right)}{\mu^{+}(F)}<\frac{\varepsilon}{3} .
\end{aligned}
$$

In conclusion

$$
\sum_{I \in D}\left|f[\mu(I)]-\ell_{1} \mu(I)\right|<\varepsilon .
$$

Clearly we can repeat this construction with $-x_{\tilde{n}}$ to find a partition $D^{*} \in \Pi(F \cap N)$ with $\delta_{\lambda}\left(D^{*}\right)<\delta(\varepsilon / 3)$ as above; again

$$
\sum_{I \in D^{*}}\left|f[\mu(I)]-\ell_{2} \mu(I)\right|<\varepsilon
$$


Hence $D \cup D^{*} \in \Pi(F)$ and

$$
\begin{gathered}
\left|\sum_{I \in D \cup D^{*}} \nu(I)-\left[\ell_{1} \mu(F \cap P)+\ell_{2} \mu(F \cap N)\right]\right| \\
=\left|\sum_{I \in D \cup D^{*}} v(I)-\mu^{+}(F)\left(\ell_{1}+\ell_{2}\right)\right| \leq 2 \varepsilon .
\end{gathered}
$$

On the other side, we can choose a decomposition $D \in$ $\Pi(F \cap P)$ with $\delta_{\lambda}(D)<\varepsilon / 2$ and then for each $I \in D$ choose symmetrically $J(I) \subset F \cap N$ with $\mu[J(I)]=-\mu(I)$ so that $D^{*}=\{J(I)\} \in \Pi(F \cap N)$.

Then we can define $D_{o}=\{I \cup J(I), I \in D\}$ to produce a decomposition of the whole $F$ with $\delta_{\lambda}\left(D_{o}\right)<\varepsilon$ but $\sum_{D_{o}} \nu(I)=$ 0 . Since $\ell_{1} \neq \ell_{2}$, the game $v$ is not $\delta_{\lambda}-B C$ integrable.

Up to this point we have been able to characterize scalar measure games and signed measure games in $\mathscr{V}$ by means of the existence of $f^{\prime}(0)$. What can be said for more general vector measure games?

We will see that differentiability at zero is not necessary, because we are considering admissible functions defined on the whole positive orthant of $\mathbb{R}^{n}$, while with $B C$ integrability we are taking into account the $\mu$-admissible directions.

For example, consider on $\mathbb{R}_{+}^{2}$ the classical example of nondifferentiable map

$$
f(x, y)= \begin{cases}\frac{x^{2} y}{x^{4}+y^{2}} & \text { whenever }(x, y) \neq \mathbf{O}, \\ 0 & \text { if }(x, y)=\mathbf{O}\end{cases}
$$

which is not differentiable on the whole orthant, being not continuous at $\mathbf{O}$. However, if $\mu \in N A^{+}$and $\eta=(\mu, \mu)$, then $R(\eta)$ reduces to a line segment and $f \circ \eta=\eta /\left(1+\eta^{2}\right) \in Q$ since it can be represented as $g \circ \mu$ with $g(t)=t /\left(1+t^{2}\right)$ which is differentiable.

Indeed the following necessary condition derives from the $B C$ integrability.

Proposition 11. Let $\mu=\left(\mu_{1}, \ldots, \mu_{n}\right)$ be in $\left(N A^{+}\right)^{n}$, and let $f$ : $\mathbb{R}_{+}^{n} \rightarrow \mathbb{R}$ be in $\mathscr{L}(\mu)$. If $f \circ \mu \in \mathscr{V}$, then $f$ admits directional derivative $d f_{\mathbf{u}}(\mathbf{O})$ along every admissible direction $\boldsymbol{u}$ (i.e., such that $\mu(F)=$ tu for some $F \in \Sigma, \mu(F) \neq 0$ and some $t>0$ ); moreover, the convergence

$$
\lim _{h \rightarrow 0} \frac{f(h \mathbf{u})}{h}=d_{\mathbf{u}} f(\mathbf{O})
$$

is uniform with respect to $\mathbf{u}$.

The proof of the existence of each directional derivative is substantially the same as that of Proposition 10, while the uniformity of the limit is deduced from the assumption of $B C$ integrability, where the defining limit

$$
\int_{E} v=\lim _{\substack{\delta_{\lambda}(D) \rightarrow 0 \\ D \in \Pi(E)}} \sum_{I \in D} v(I)
$$

is uniform with respect to $E \in \Sigma$.
Observe that the requirement $f \in \mathscr{L}(\mu)$ in the previous statement could be weakened by requiring that for each radial direction $\mathbf{u}$ that crosses $\partial R(\mu)$ at a point $P \neq \mathbf{O}$, the ratios $f(t \mathbf{u}) / t$ are bounded in $] 0,1]$.

Unfortunately, even with this weakening, the condition expressed in the above Proposition does not characterize vector measure games in $B C$. To get convinced, we present the following example.

Example 12. Consider $f(x, y)$ as above which is not differentiable at $\mathbf{O}$, since it is not continuous, despite the fact that it admits directional derivative at every direction $\mathbf{u}$ on the positive orthant, given by

$$
d_{\mathbf{u}} f(\mathbf{O})= \begin{cases}\frac{\cos ^{2} \vartheta}{\sin \vartheta} & \text { if } \sin \vartheta \neq 0, \\ 0 & \text { if } \sin \vartheta=0\end{cases}
$$

where $\mathbf{u}=(\cos \vartheta, \sin \vartheta)$.

A simple computation in fact provides, for $\sin \vartheta \neq 0$,

$$
\left|\frac{f(t \cos \vartheta, t \sin \vartheta)}{t}-d_{\mathbf{u}} f(\mathbf{O})\right|=\frac{t^{4} \cos ^{6} \vartheta}{|\sin \vartheta|\left(t^{4} \cos ^{4} \vartheta+\sin ^{2} \vartheta\right)}
$$

For the sake of simplicity set $\alpha=\sin \vartheta, \beta=\cos ^{2} \vartheta$. Then we want to show that the ratios $\beta^{3} t^{4} /|\alpha|\left(t^{4} \beta^{2}+\alpha^{2}\right)$ can be made arbitrarily small, somehow independently on the values of $\alpha, \beta$.

Easily

$$
\frac{\beta^{3} t^{4}}{|\alpha|\left(t^{4} \beta^{2}+\alpha^{2}\right)} \leq \frac{\beta^{3} t^{4}}{|\alpha|^{3}}
$$

which in turn is smaller than $\varepsilon$ for $t^{4}<\varepsilon \cdot\left(|\alpha|^{3} / \beta^{3}\right)=$ $\varepsilon\left(|\sin \vartheta|^{3} / \cos ^{6} \vartheta\right)$; one immediately checks that the map $\vartheta \rightarrow$ $|\sin \vartheta|^{3} / \cos ^{6} \vartheta$ is increasing for $0<\vartheta<\pi / 2$.

Consider now $\gamma:[0,1] \rightarrow \mathbb{R}$ defined as

$$
\gamma(x)= \begin{cases}x^{2} & \text { for } 0 \leq x \leq x_{o} \\ 2 x_{o} x-x_{o}^{2} & \text { for } x>x_{o}\end{cases}
$$

with $0<x_{o}<1$.

Then consider the "reverse" function $G:[0,1] \rightarrow \mathbb{R}$ defined as $G(x)=1-\gamma(1-x)$; then the subset of $\mathbb{R}_{+}^{2}$ given by $\{(x, y), x \in[0,1], \gamma(x) \leq y \leq G(x)\}$ is a zonoid, that is, the range of a nonatomic measure $\mu$ (see [9]), and the admissible directions for such $\mu$ have slopes not exceeding $2 x_{o}$; this is enough to achieve the required uniformity for the vector measure game $f \circ \mu$.

We will now prove that the weakened assumption is satisfied.

Fix an admissible direction $\mathbf{u}=\left(u_{1}, u_{2}\right)$, that is, such that span $\mathbf{u}$ intersects $\partial R(\mu)$ at a point $P \neq \mathbf{O}$.

Observe then that all the ratios along the $\mathbf{u}$ direction are bounded.

However the game is not $B C$ integrable; in fact, let $F$ be a set for which $\mu(F)=\left(x_{o}, x_{o}^{2}\right)$; whichever $\delta>0, \delta<$ 
$x_{o} / 3$ we choose, we can always find a subset $I$ of $F$ such that $\mu(I)=\left(\delta, \delta^{2}\right)$ (thanks to the Hereditarily overlapping boundary property [10]).

Now one can always decompose $F \backslash I$ into $n$ sets $J_{1}, \ldots, J_{n}$ in such a way that $\mu\left(J_{k}\right)=m(F \backslash I) / n<\delta, k=1, \ldots, n$.

Thus, as $\mu\left(J_{k}\right)=(1 / n)\left(x_{o}-\delta, x_{o}^{2}-\delta^{2}\right)$, one finds

$$
\begin{aligned}
(f \circ \mu)\left(J_{k}\right)= & f\left(\frac{x_{o}-\delta, x_{o}^{2}-\delta^{2}}{n}\right) \\
= & \frac{\left(x_{o}-\delta\right)^{3}\left(x_{o}+\delta\right)}{n^{3}} \\
& \times\left(\frac{\left(x_{o}-\delta\right)^{4}}{n^{4}}+\frac{\left(x_{o}-\delta\right)^{2}\left(x_{o}+\delta\right)^{2}}{n^{2}}\right)^{-1} .
\end{aligned}
$$

Consider then $D=\left\{I, J_{1}, \ldots, J_{n}\right\} \in \Pi(F)$; we have

$$
\begin{aligned}
\sum_{F_{i} \in D}(f \circ \mu)\left(F_{i}\right)= & (f \circ \mu)(I)+n(f \circ \mu)\left(J_{1}\right) \\
= & \frac{1}{2}+\frac{\left(x_{o}-\delta\right)^{3}\left(x_{o}+\delta\right)}{n^{2}} \\
& \times\left(\frac{\left(x_{o}-\delta\right)^{4}}{n^{4}}+\frac{\left(x_{o}-\delta\right)^{2}\left(x_{o}+\delta\right)^{2}}{n^{2}}\right)^{-1} \\
= & \frac{1}{2}+\frac{x_{o}^{2}-\delta^{2}}{\left(x_{o}+\delta\right)^{2}+\left(\left(x_{o}-\delta\right)^{2} / n^{2}\right)}
\end{aligned}
$$

Hence, letting $n \rightarrow \infty$ we have that $\sum_{F_{i} \in D}(f \circ \mu)\left(F_{i}\right)$ can be made strictly greater than 1 .

On the other side, we can decompose $F$ into finitely many sets $F_{1}, \ldots, F_{n}$ each having $\mu\left(F_{j}\right)=\mu(F) / n$ and $n$ large enough to have $\mu\left(F_{j}\right)<\delta$. On the decompositions of this type then

$$
\begin{aligned}
\sum_{D}(f \circ \mu)\left(F_{j}\right) & =n(f \circ \mu)\left(F_{1}\right) \\
& =n f\left(\frac{x_{o}}{n}, \frac{x_{o}^{2}}{n}\right)=n \frac{x_{o}^{4} / n^{3}}{x_{o}^{4} / n^{4}+x_{o}^{4} / n^{2}} \\
& =\frac{1}{1+1 / n^{2}} \longrightarrow 1^{-} .
\end{aligned}
$$

Therefore, the $B C$ integral does not exist.

One may be interested also in the naturally arising space $\mathscr{V}_{\infty}$ of games obtained as $\|\cdot\|_{\infty}$-limit of sequences of games in $\mathscr{V}$.

Several questions arise in this space; the first open one is Are games in $\mathscr{V}_{\infty}$ still vector measure games?

And even in the negative, can one at least characterize those vector measure games in terms of properties of the function $f$ that defines them?

It is indeed rather difficult to characterize the $\|\cdot\|_{\infty^{-}}$ closure of $\mathscr{V}$, because we could not reach so far any satisfactory result relative to $\|\cdot\|_{\infty}$-convergence; to be more precise given an admissible function $f$ on $\mathbb{R}_{+}^{n}$ and a sequence of vector measures $\left(\mu_{k}\right)_{k} \subset\left(N A^{+}\right)^{n}$, which convergence of $\left(\mu_{k}\right)_{k}$ ensures that the sequence $f \circ \mu_{k} \stackrel{\|\cdot\|_{\infty}}{\longrightarrow} f \circ \mu$ ?

And, dually, one can also ask: given a sequence of admissible functions $f_{k}: \mathbb{R}_{+}^{n} \rightarrow \mathbb{R}$ and a vector measure $\mu$, what kind of convergence of the sequence $\left(f_{k}\right)_{k}$ ensures that the sequence of games $f_{k} \circ \mu \stackrel{\|\cdot\|_{\infty}}{\longrightarrow} f \circ \mu$ ?

We can give so far only a sufficient condition; to this extent we first set the following structure on the already defined space $\mathscr{A}$; set

$$
\begin{aligned}
& d_{\text {lip }}(f, g) \\
& =\inf \{L>0 \text {, such that } L \text { is a Lipschitz constant for } \\
& \quad f-g \text { in the increasing directions }\} .
\end{aligned}
$$

Then $d_{\text {lip }}$ defines a metric on $\mathscr{A}$. The analogous $d_{\text {lip }}$ is a semimetric on each $\mathscr{L}(\mu)$.

Then we have the following.

Proposition 13. Let $\mu \in\left(N A^{+}\right)^{n}$ and let $f$ be admissible. If $f$ is a $d_{\text {lip }}$ cluster point for $\mathscr{L}(\mu)$, then $f \circ \mu \in \mathscr{V}_{\infty}$.

Proof. Fix $\varepsilon>0$, and choose $\sigma=\varepsilon / 4$; set $\delta=\sigma /\left(\mu_{1}(\Omega)+\cdots+\right.$ $\mu_{n}(\Omega)$ ). Next, let $g \in \mathscr{L}^{\prime}(\mu)$ (the set of differentiable functions in $\mathscr{L}(\mu))$ with $d_{\text {lip }}(f, g)<\delta$.

This means that $f-g$ is Lipschitz in the $\mu$-link directions with constant $L<\delta$.

Fix now $F \in \Sigma, D \in \Pi(F), T_{D} \in \mathscr{T}_{D}$; for the game ( $f \circ$ $\mu)-(g \circ \mu)$ one computes

$$
\begin{aligned}
& \left|S\left[(f \circ \mu)-(g \circ \mu), D, T_{D}\right]\right| \\
& \leq \sum_{i}\left|[(f-g) \circ \mu]\left(D_{i} \cup T_{i}\right)-[(f-g) \circ \mu]\left(T_{i}\right)\right| \\
& <\delta \sum_{i}\left\|\mu\left(D_{i} \cup T_{i}\right)-\mu\left(T_{i}\right)\right\| \\
& =\delta \sum_{i}\left\|\mu\left(D_{i}\right)\right\|=\delta\left[\mu_{1}(F)+\cdots+\mu_{n}(F)\right] \leq \sigma
\end{aligned}
$$

from which we deduce $\|(f \circ \mu)-(g \circ \mu)\|_{\infty} \leq 4 \sigma=\varepsilon$. Since $g \in \mathscr{L}^{\prime}(\mu)$ the game $g \circ \mu \in \mathscr{V}$ thanks to Proposition 3 in [5], and the proof is complete.

\section{Conflict of Interests}

The authors declare that there is no conflict of interests regarding the publication of this paper.

\section{References}

[1] R. J. Aumann and L. S. Shapley, Values of Non-Atomic Games, Princeton University Press, Princeton, NJ, USA, 1974.

[2] Y. Tauman, "A characterization of vector measure games in pNA," Israel Journal of Mathematics, vol. 43, no. 1, pp. 75-96, 1982. 
[3] I. Milchtaich, "Vector measure games based on measures with values in an infinite-dimensional vector space," Games and Economic Behavior, vol. 24, no. 1-2, pp. 25-46, 1998.

[4] A. Neyman, "Values of games with infinitely many players," in Handbook of Game Theory, R. Aumann and S. Hart, Eds., vol. 3, pp. 2121-2167, North Holland, Amsterdam, The Netherlands, 2002.

[5] F. Centrone and A. Martellotti, "The Burkill-Cesari integral on spaces of absolutely continuous games," International Journal of Mathematics and Mathematical Sciences, vol. 2014, Article ID 659814, 9 pages, 2014.

[6] F. Centrone and A. Martellotti, "A mesh-based notion of differential for TU games," Journal of Mathematical Analysis and Applications, vol. 389, no. 2, pp. 1323-1343, 2012.

[7] D. Monderer, "A Milnor condition for nonatomic Lipschitz games and its applications," Mathematics of Operations Research, vol. 15, no. 4, pp. 714-723, 1990.

[8] L. Cesari, "Quasi-additive set functions and the concept of integral over a variety," Transactions of the American Mathematical Society, vol. 102, pp. 94-113, 1962.

[9] D. Candeloro and A. Martellotti, "Geometric properties of the range of two-dimensional quasi-measures with respect to the Radon-Nikodym property," Advances in Mathematics, vol. 93, no. 1, pp. 9-24, 1992.

[10] A. Martellotti, "Countably additive restrictions of vector-valued quasi measures with respect to range preservation," Bollettino della Unione Matematica Italiana. Sezione B. Serie VII, vol. 2, no. 2, pp. 445-458, 1988. 


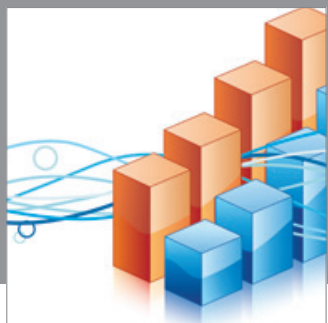

Advances in

Operations Research

mansans

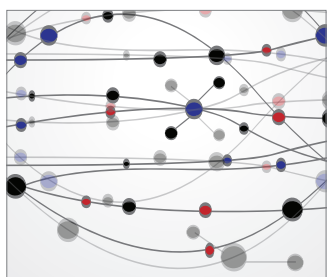

The Scientific World Journal
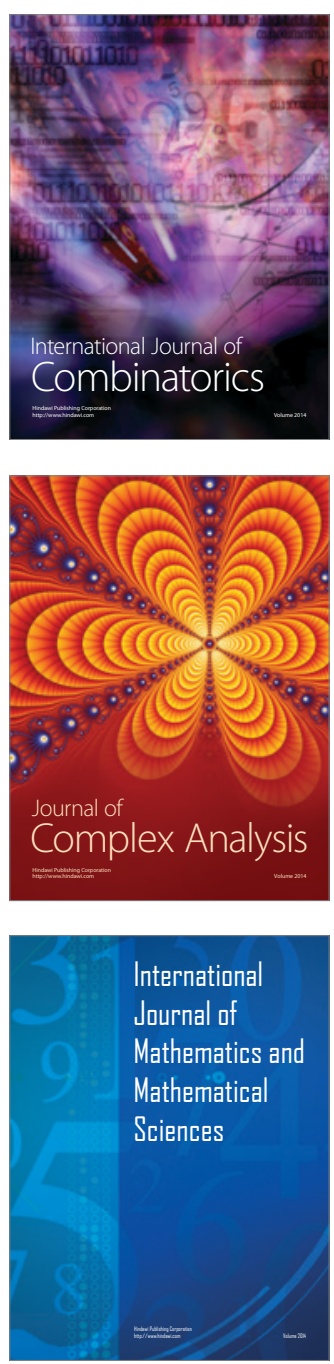
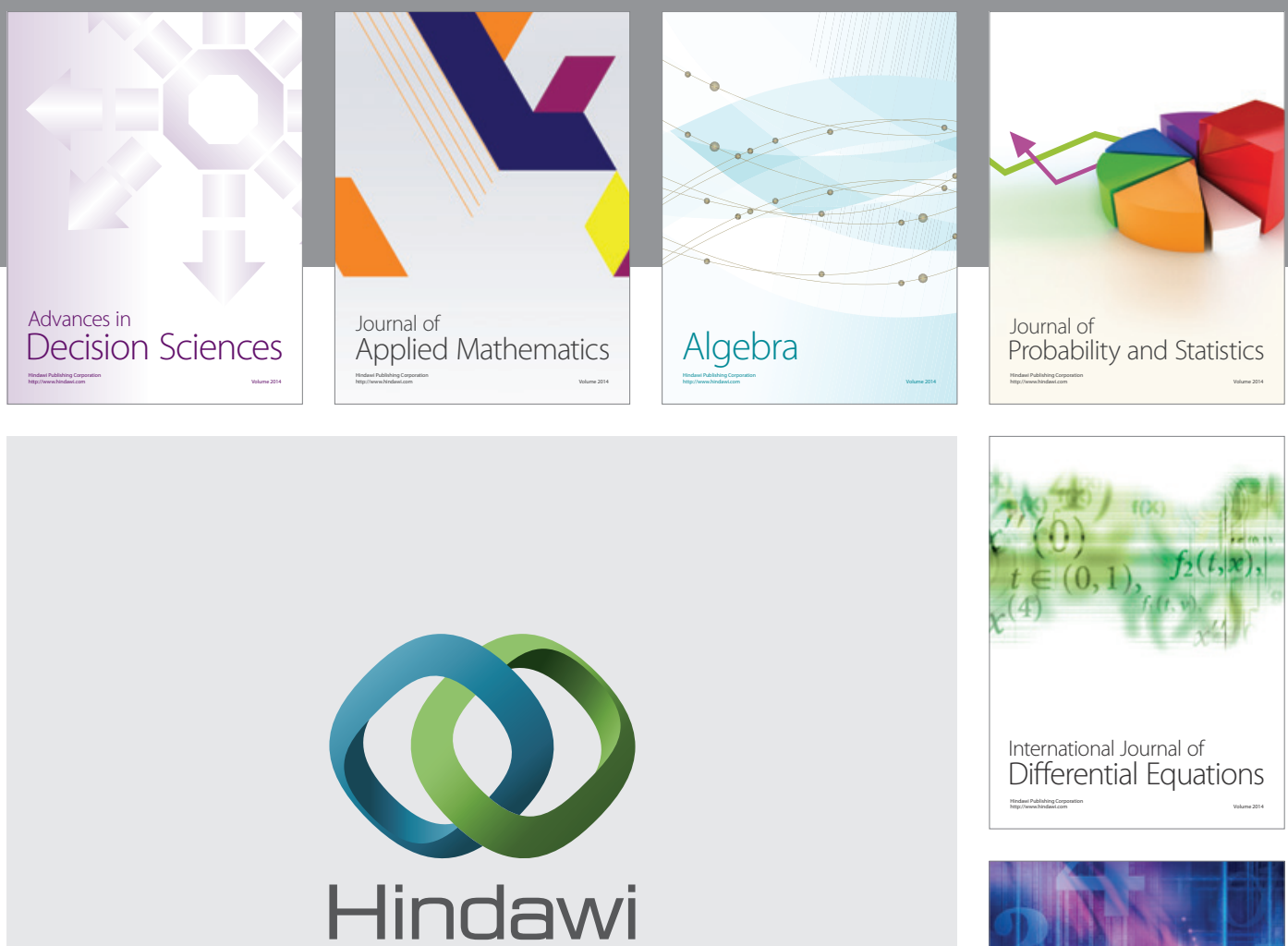

Submit your manuscripts at http://www.hindawi.com
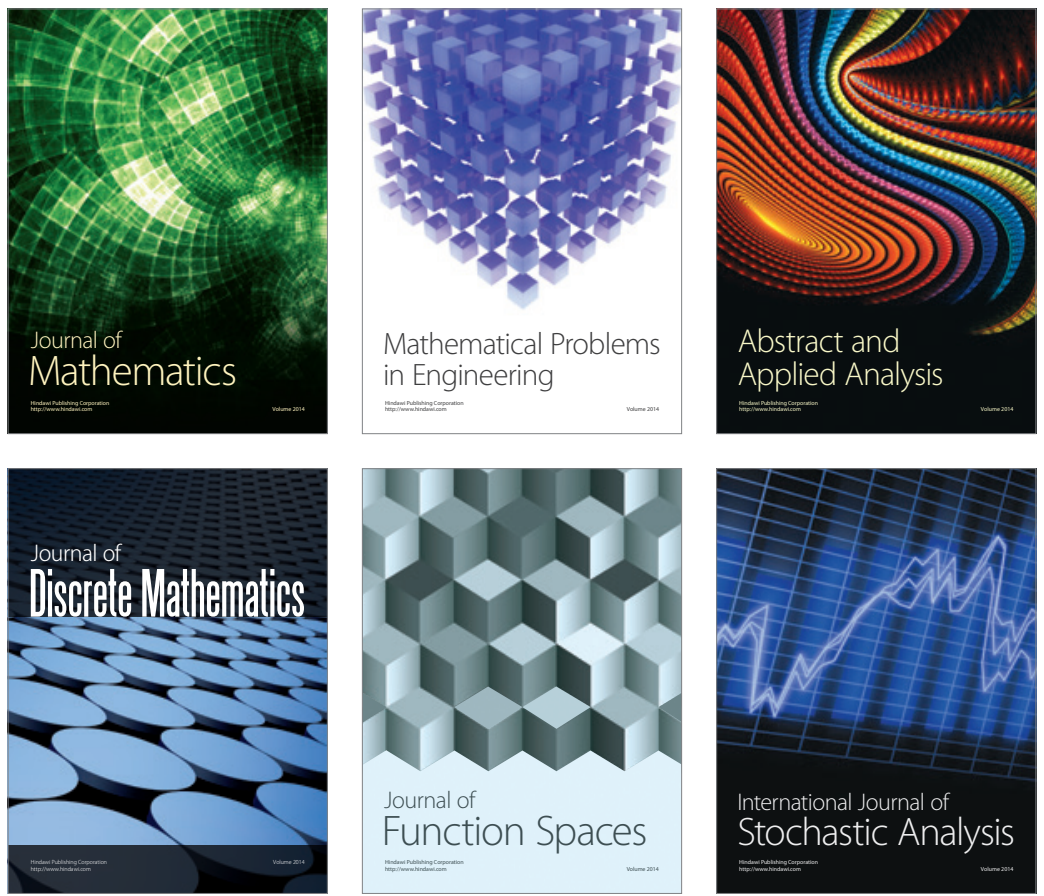

Journal of

Function Spaces

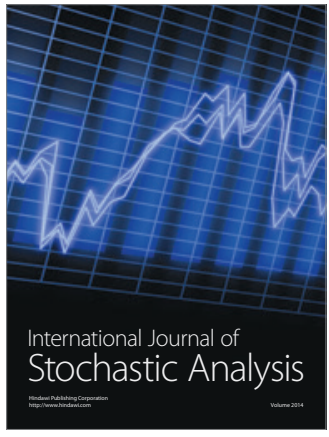

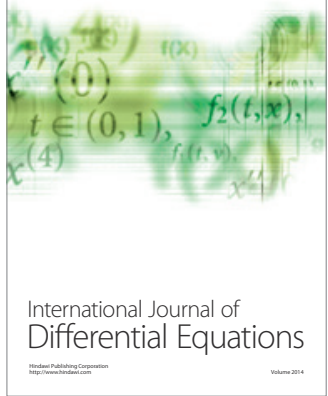
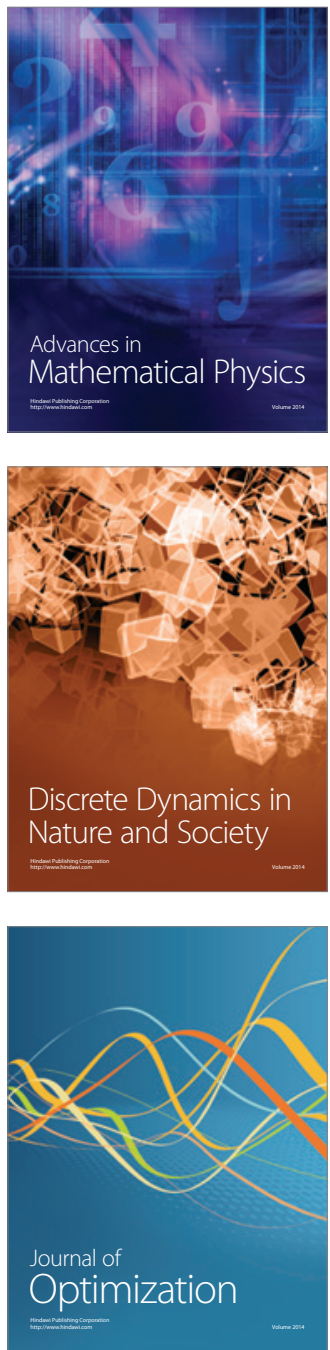